\title{
Listas de verificación para la elaboración, tutorización y evaluación de Trabajos Fin de Grado \\ Bachelor's Degree Final Project Checklists for elaboration, tutoring, and evaluation.
}

\author{
Ana María Martín-Nogueras, Beatriz María Bermejo-Gil, Raquel Diez-García, Emérita García-Astudillo, Inés \\ Llamas-Ramos, Natalia Montes-Carrasco, Carlos Moreno-Pascual, Carmen Oreja-Sánchez, Jesús Orejuela-Rodríguez, \\ Fátima Pérez-Robledo, Juan Luis Sánchez-González \\ Email: anamar@usal.es, Beatriz.Bermejo@usal.es, raquel10@usal.es,00774612@usal.es, inesllamas@usal.es, \\ natmontes@usal.es, moreno@usal.es, carmenoreja@usal.es, jorejes@usal.es, fatima_pr@usal.es, juanluissanchez@usal.es.
}

\author{
Departamento de Enfermería y Fisioterapia \\ Universidad de Salamanca \\ Salamanca, España
}

\begin{abstract}
Resumen. El Trabajo Fin de Grado (TFG) es una asignatura obligatoria en los títulos de Grado. La diversidad existente en el tipo de trabajo y la falta de mecanismos de coordinación originan disparidad de criterios en la elaboración-tutorización y evaluacióncalificación de estos. El objetivo de este trabajo fue diseñar un instrumento que facilitase la realización, tutorización y evaluación de los TFG en la titulación de Grado en Fisioterapia en una universidad pública española. Se diseñaron 6 listas de verificación, una común que incluía las normas de estilo, extensión y estructura y otras 5 para los tipos de TFG contemplados: trabajo de investigación, revisión bibliográfica sistemática, proyectos de investigación, trabajo profesional (caso clínico y protocolo de intervención). Los apartados de las listas y sus epígrafes fueron consensuados entre un equipo de 11 profesores de la titulación. Las listas fueron distribuidas durante el segundo cuatrimestre de 2021 entre profesores y estudiantes de $4^{\circ}$ curso. Disponer de listas de verificación organizadas por apartados y epígrafes facilita la elaboración, tutorización y evaluación de los TFG, minimiza la aparición de errores durante las mismas y resultan instrumentos fácilmente exportables a otras universidades y titulaciones en el ámbito de las Ciencias de la Salud.
\end{abstract}

Palabras clave: Trabajo fin de Grado, Lista verificación, Grado de Fisioterapia

Abstract. The Bachelor's Degree Final Project (BDFP) is a
compulsory subject for undergraduate university students. Due to the
different types of works and the lack of coordination mechanism,
there is a disparity of criteria in the preparation-tutoring and
evaluation-qualification of them. The aim of this work was to design
an instrument that facilitates the elaboration, tutoring, and evaluation
of the TFG in the Physiotherapy Bachelor's degree at a spanish
public university. A bank of six checklists was designed; a common
one that included the rules of style, extension, and structure; and
another five regarding each specific type of BDFP: research work,
systematic bibliographic review, research projects, and professional
work (clinical case or intervention protocol). A team of 11 university
professors agreed and designed the sections and headings of each
checklist. A final version was distributed during the second semester
of 2021 among teachers and 4th-year students. It is remarkable that
having organized, and specific checklists facilitate the preparation,
tutoring, and evaluation of the BDFP, assuring the most important task, consistency as well as minimizing mistakes. Furthermore, they are easily exportable tools to other universities and health sciences related degrees.

\section{Keywords: Bachelor's Degree Final Project; checklists; Physiotherapy Degree.}

\section{INTRODUCCIÓN}

El Trabajo Fin de Grado (TFG) se incorporó como una asignatura obligatoria en los títulos de Graduado tras la adaptación de las titulaciones al Espacio Europeo de Educación Superior (EEES) a partir del año 2009.

La normativa nacional no recoge aspectos específicos respecto a la elaboración o diseño de los TFG, de forma que cada titulación, centro y universidad ha desarrollado sus normativas específicas para la elaboración, presentación y defensa de estos trabajos. Si bien, en general son instrucciones escuetas o simplemente de formato o procedimiento.

Cada titulación suele recoger un conjunto de diferentes tipos de trabajo y en ocasiones con diferentes estructuras. Esta diversidad, y la falta de mecanismos de coordinación entre tutores y evaluadores dentro de una misma titulación, origina disparidad de criterios en la elaboración-tutorización, evaluación-calificación de los trabajos, así como incumplimiento en las expectativas de los estudiantes (Moreno, Jaén, y Ruiz 2019).

El objetivo de este trabajo fue diseñar un instrumento que facilitase la realización, tutorización y evaluación de los TFG en la titulación de Grado en Fisioterapia en una universidad pública española.

Las mejoras esperables con el desarrollo del presente proyecto fueron:

- Conseguir una mayor autonomía de los estudiantes a la hora de desarrollar sus TFG, así como la posibilidad de llevar a cabo sus propias autoevaluaciones. 
- Facilitar la tutorización de los profesores durante el proceso de elaboración del TFG.

- Minimizar los errores que por despiste pueden aparecer en el formato o contenido de los TFG, mejorando, por tanto, el rendimiento académico de los estudiantes.

- Facilitar el proceso de evaluación de los profesores miembros de las comisiones de evaluación al objetivar de forma pormenorizada y conjunta los diferentes aspectos del TFG.

- Homogeneizar el proceso de evaluación individual y colectiva, y la posible calificación final por parte de los profesores de la comisión evaluadora.

\section{Contexto}

Este trabajo se enmarca en el contexto de la titulación de Grado en Fisioterapia, de una universidad pública española.

El TFG se incorpora como una asignatura en los títulos de grado tras la adaptación de las titulaciones al EESS, siendo un requisito obligatorio para la obtención del título. Consiste en un trabajo académico original de diferente naturaleza, según la titulación, tutorizado y supervisado por un profesor tutor y que finalmente debe ser presentado y defendido ante un tribunal universitario.

Normativamente el TFG está regulado por el Real Decreto 1393/2007 (RDL 1393/2007, de 30 de septiembre), por el que se establecen la ordenación de las enseñanzas universitarias oficiales, donde en su artículo 12.3, dedicado a las directrices para el diseño de los títulos de Graduado, incluye "estas enseñanzas concluirán con la elaboración y defensa de un trabajo fin de grado». En ese mismo artículo y en el punto 7 se añade que "el trabajo de fin de grado tendrá un mínimo de 6 créditos y un máximo de 12,5 por ciento del total de los créditos del título, deberá realizarse en la fase final del plan de estudios y estar orientado a la evaluación de las competencias asociadas al título". Las competencias de los grados universitarios que dan acceso a profesiones reguladas están recogidas en las ordenes CIN correspondientes, en el caso del Grado de Fisioterapia, ORDEN CIN/2135/2008, de 3 de julio (Orden CIN/2135/2008, de 3 de julio). En ella se establece en el módulo de Prácticas tuteladas y Trabajo Fin de Grado que las competencias a alcanzar son la "presentación y defensa ante el Tribunal universitario de un proyecto de fin de grado, consistente en un ejercicio de integración de los contenidos formativos recibidos y las competencias adquiridas".

Otra normativa que hace referencia al TFG es el Estatuto del Estudiante Universitario, RD 1791/2010, de 30 de diciembre (RDL 1791/2010, de 31 de diciembre), donde se recoge que los estudiantes tienen derecho "a contar con tutela efectiva, académica y profesional, en el trabajo fin de grado y, en su caso, en las prácticas externas que se prevean en el plan de estudios" y "a contar con el reconocimiento y protección de la propiedad intelectual del trabajo fin de grado y de los trabajos previos de investigación en los términos que se establecen en la legislación vigente sobre la materia"; finalmente en su artículo 27 establece que "los proyectos de fin de carrera, trabajos de fin de grado y máster, así como las tesis doctorales, se regirán por su normativa específica".

Por lo tanto, la normativa no recoge disposiciones sobre el tipo de trabajo o cómo debe elaborarse o evaluarse, de forma que cada universidad, centro y título han aplicado su propio criterio. No se ha encontrado ningún consenso entre universidades o titulaciones, tan sólo guías diversas de cómo llevar a cabo un TFG, las cuales son muy diversas en extensión y contenido. Nuestra universidad dispone de un reglamento tipo que cada titulación y centro ha desarrollado de forma específica, en nuestro caso se encuentra disponible en la página web del centro (https://enfermeriayfisioterapia.usal.es/trabajos-de-fin-degrado/).

La asignatura de TFG en la que se encuadra este trabajo tiene asignados 6 ECTS en el Plan de Estudios de Grado en Fisioterapia y está contemplado en el segundo semestre de $4^{\circ}$ curso. Nuestro reglamento establece que el contenido del trabajo debe corresponder a uno de los siguientes tipos: trabajos de investigación, de revisión bibliográfica sistemática, de carácter profesional $\mathrm{o}$ proyectos de investigación; incluyéndose además las instrucciones sobre normas de estilo, extensión y estructura.

La incorporación de los TFG a la titulación de Fisioterapia, al igual que en otras muchas titulaciones, se hizo sin existir una tradición previa en este tipo de trabajos y sin que los profesores contasen con formación específica para ello. A lo largo de estos años los profesores han ido aprendiendo a desarrollar su labor como tutores y evaluadores en las correspondientes comisiones. Desde las vivencias y experiencias acumuladas a partir de la tutorización y evaluación a lo largo de estos últimos años es de donde surge este trabajo, donde un equipo de profesores del Grado en Fisioterapia identificaron un conjunto de dificultades, en estudiantes y profesores, durante el proceso de elaboracióntutorización y evaluación-calificación de los TFG que justificaron la elaboración de este proyecto, el cual fue presentado a la convocatoria 2020 del programa de innovación docente de la universidad de origen; obteniendo una puntuación de 84/100 junto a una financiación para la difusión de los resultados.

El objetivo general fue diseñar un instrumento que facilitase la realización, tutorización y evaluación de los TFG en la titulación de Grado en Fisioterapia en una universidad pública española. Como objetivos específicos se establecieron: 1 . Elaborar listas de verificación con la estructura que deben presentar cada tipo de TFG contemplado en la titulación a partir del consenso sobre los apartados, subapartados y contenidos obligatorios y optativos que cada tipo de trabajo debía contener; 2. Elaborar una lista de verificación sobre aspectos formales de los documentos de presentación de los TFG; 3. Presentar y remitir las listas de verificación a los diferentes profesores y estudiantes del Grado en Fisioterapia para su uso voluntario durante el proceso de realización del TFG en el Grado de Fisioterapia en el curso 2020-21; 4. Evaluar el grado de uso y utilidad de los instrumentos diseñados.

\section{DESCRIPCIÓN}

El proyecto fue diseñado para llevarse a cabo en dos etapas diferenciadas. Una primera etapa dedicada a la elaboración de los instrumentos (septiembre 2020-enero 2021) y otra la de aplicación y evaluación de éstos (febrero 2021-julio 2021). 
En el mes de septiembre de 2020 se estableció una reunión de coordinación del equipo de trabajo en la que se establecieron las directrices de trabajo y el cronograma a seguir. Se programaron un total de 6 sesiones de trabajo de 2 horas de duración, una por cada lista de verificación que se elaboró. Dada la situación actual de pandemia todas las sesiones se realizaron de forma síncrona mediante videoconferencia por Google Meet. La forma de trabajo en cada sesión fue similar, una semana antes de la reunión la coordinadora del proyecto distribuía entre el resto del equipo un borrador o plantilla sobre los aspectos a discutir y valorar en cada una de las listas de verificación, atendiendo a las instrucciones ofrecidas por la dirección del Centro en su página web (https://enfermeriayfisioterapia.usal.es/trabajosde-fin-de-grado/) y a las recomendaciones de la Red Equator (The EQUATOR Network, 2021) para el tipo de trabajo contemplado. Todas las cuestiones y epígrafes incluidos en cada una de las listas de verificación fueron resueltas por consenso entre los miembros del equipo.

Durante el mes de febrero de 2021 las listas de verificación fueron distribuidas mediante un mensaje de correo electrónico desde el decanato del centro, a todos los profesores de la titulación y estudiantes de $4^{\circ}$ curso del Grado de Fisioterapia para su utilización durante la asignatura de TFG, de segundo cuatrimestre. Durante esta segunda fase, y en una nueva sesión de trabajo, el equipo de profesores diseñó una encuesta para ser distribuida entre profesores y estudiantes a través de Google Forms y que permitiría conocer el grado de uso y utilidad de las listas elaboradas (https://forms.gle/qiLHsMU7gumtVpQr5)

\section{Resultados}

Se elaboraron un total de 6 listas de verificación (https://sites.google.com/usal.es/listas-tfg), una genérica para todos los tipos de TFG y que contemplaba las Normas de estilo, extensión y estructura del TFG y otras 5 para los tipos de trabajo contemplados por el centro: TFG trabajo de investigación, TFG revisión bibliográfica sistemática, TFG proyectos de investigación, TFG trabajo profesional (caso clínico), TFG trabajo profesional (protocolo de intervención). En el caso de los TFG profesionales se consideraron los dos tipos más habituales, teniendo en cuenta que son un tipo de trabajo muy diverso y que supone una alta dificultad a la hora de estandarizar los epígrafes.

El formato de todas las listas fue unificado en forma de columnas, en la primera de ellas se incluyó cada uno de los apartados y cuestiones a valorar o tener en cuenta, en la segunda se incluyó un sistema de casillas de verificación para activar, la tercera incluía observaciones que el equipo consideró apropiadas y la cuarta un espacio para que el tutor o evaluador incluyese sus comentarios.

Así la lista de Normas de estilo, extensión y estructura del TFG incluyó los apartados de: formato y normas de estilo, portada, índice, resumen, tablas, figuras y gráficos, bibliografía y otros. La lista de TFG trabajo de investigación incluía en columnas distintos tipos de estudios (experimentales, descriptivos trasversales y descriptivos longitudinales) con los apartados de introducción, objetivos, material y métodos, resultados, discusión y conclusiones. La lista TFG revisión bibliográfica sistemática incluyó los apartados de introducción, objetivos, estrategia de búsqueda y selección de estudios, síntesis y análisis de resultados, discusión y conclusiones. La de TFG proyectos de investigación los de introducción, objetivos, material y métodos y plan de trabajo. Los TFG trabajo profesional (caso clínico y protocolo de intervención) quedaron estructurados en introducción, objetivo, desarrollo del tema y conclusiones.

Estas listas fueron puestas a disposición de la comunidad universitaria en formato PDF a través de la página web elaborada por el equipo del proyecto (https://sites.google.com/usal.es/listas-tfg).

La encuesta sobre el uso y utilidad de las listas fue distribuida entre profesores de la titulación y estudiantes de cuarto curso, el 25 de junio de 2021 a través del decanato del centro, manteniéndose abierta hasta el 12 de julio de 2021. La población a la que iba dirigida era aproximadamente de 110 personas (60 profesores y 50 estudiantes).

Se recogieron un total de 36 respuestas de las cuales 24 $(66,7 \%)$ fueron estudiantes y el resto profesores. Un $13,9 \%$ de los encuestados manifestaron no haber utilizado las listas de verificación porque no habían recibido información sobre ellas y un $2,8 \%$ por no haber tutorizado ningún TFG durante el curso. La lista más utilizada, como era de esperar, fue la de Normas de estilo, extensión y estructura, en el 70\% de los que las habían utilizado. Entre las propias de los tipos de trabajos, las de TFG de revisión sistemática $(56,7 \%)$ y TFG de investigación $(30 \%)$ fueron las que mayor porcentaje de uso alcanzaron (Figura 1). Respecto al uso y utilidad en el grupo de los estudiantes, el $89,5 \%$ estaban de acuerdo o muy de acuerdo en que las listas han sido útiles y les han facilitado el trabajo y el $52,6 \%$ consideró que les habían ayudado a un mejor resultado. Tan solo el 10,5\% cambiaría el diseño y nadie consideró que fueran incompletas. En el grupo de profesores, el $81,8 \%$ estaba de acuerdo o muy de acuerdo con que fueron útiles y que habían facilitado el trabajo. Tan sólo un profesor consideró que cambiaría el diseño y que eran incompletas. El $94,7 \%$ de los estudiantes y el $81,8 \%$ de los profesores manifestaron que las volverían a utilizar.

Finalmente, en el grado de satisfacción general en la utilización de las listas se obtuvo una media de 8,4 puntos sobre 10 (DT 0,895 ) en el grupo de los estudiantes, con un rango entre 7 y 10 y de 9,6 puntos sobre 10 (DT 0,674 ) en el de profesores, con un rango entre 8 y 10 puntos (Figura 2).

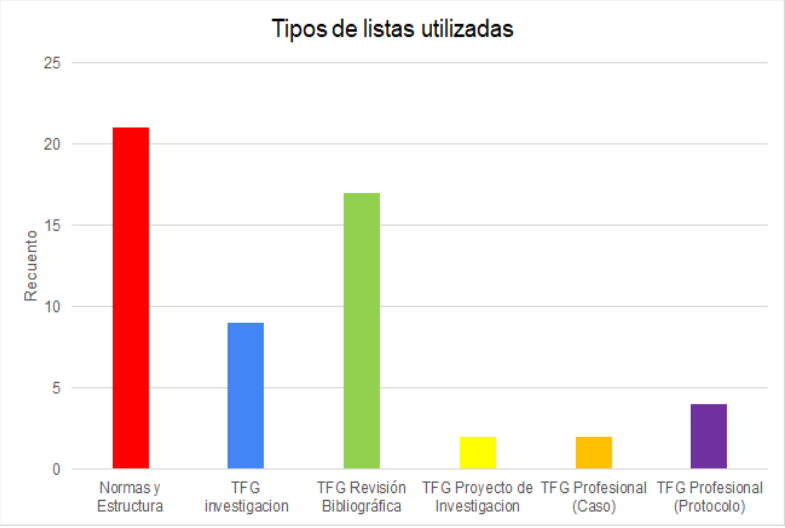

Figura 1 


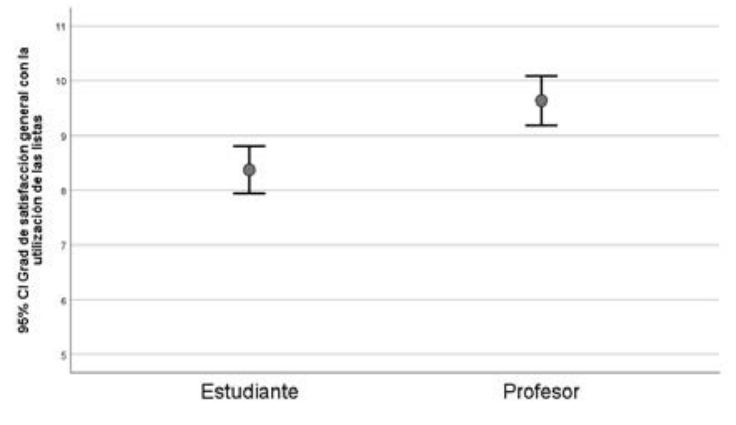

Figura 2

\section{CONCLUSIONES}

Ha sido posible establecer, mediante consenso entre profesores, listas de verificación que facilitan la elaboración y evaluación de los TFG al permitir verificar los apartados, contenidos y aspectos formales que se esperan en cada tipo de TFG contemplado en la normativa de nuestro Centro.

Disponer de listas de verificación organizadas por apartados y epígrafes presumiblemente minimiza la aparición de errores en la elaboración y el resultado del TFG.

Las listas elaboradas son fácilmente exportables al título de Grado de Fisioterapia de otras universidades, así como adaptadas a otras titulaciones en el ámbito de las Ciencias de la Salud.

\section{REFERENCIAS}

Encuesta PROYECTO DE INNOVACIÓN. Google Docs. (27 de julio de 2021). Listas de verificación TFG. https://forms.gle/qiLHsMU7gumtVpQr5

Orden CIN/2135/2008, de 3 de julio, por la que se establecen los requisitos para la verificación de los títulos universitarios oficiales que habiliten para el ejercicio de la profesión de Fisioterapeuta (2008). Boletín Oficial del Estado 174, de19 de julio de 2008, 31684 a 31687. https://www.boe.es/boe/dias/2008/07/19/pdfs/A3168431687.pdf.

Listas verificación TFG. (27 de julio de 2021). Página web del Proyecto de innovación docente ID 2020/010 Usal: "Elaboración de listas de verificación para la realización, tutorización de Trabajos Fin de Grado". https://sites.google.com/usal.es/listas-tfg.

Real Decreto 1393/2007, de 29 de octubre, por el que se establece la ordenación de las enseñanzas universitarias oficiales (2007) Boletín Oficial del Estado 260, de 30 de octubre de 2007. https://www.boe.es/buscar/pdf/2007/BOE-A-200718770-consolidado.pdf.

Real Decreto 1791/2010, de 30 de diciembre, por el que se aprueba el Estatuto del Estudiante Universitario (2010) Boletín Oficial del Estado 318, de 31 de diciembre de 2010. https://www.boe.es/buscar/pdf/2010/BOE-A2010-20147-consolidado.pdf.

Resources in Spanish/Recursos en español. The EQUATOR Network. (Sin fecha) Recuperado de https://www.equator-network.org/library/spanishresources-recursos-en-espanol/-4634201945200829.

Rodríguez Moreno, J., Molina Jaén M.D. y Colmenero Ruiz M.J. (2019). La voz de los estudiantes de la titulación de Educación Primaria sobre el Trabajo Fin de Grado. Educação e Pesquisa 45, e200829. doi: 10.1590/S16784634201945200829.

Trabajo Fin de Grado. (27 de julio de 2021). Facultad de Enfermería y fisioterapia Universidad de Salamanca https://enfermeriayfisioterapia.usal.es/trabajos-de-fin-degrado/. 